Article

\title{
Modified Bentonite Clay in UV-Curable Coating Applications
}

\author{
Nantana Jiratumnuku1 ${ }^{1,2, *}$, Patcharee Manowanna ${ }^{1,2}$, and Narangkhana Premmag ${ }^{1,2}$ \\ 1 Department of Materials Science, Faculty of Science, Chulalongkorn University, Bangkok 10330, Thailand \\ 2 National Center of Excellence for Petroleum, Petrochemicals and Advanced Materials, Chulalongkorn \\ University, Bangkok 10330, Thailand \\ E-mail: jnantana@sc.chula.ac.th*
}

\begin{abstract}
Modified bentonite clay was prepared by cationic exchange between the $\mathrm{Na}+$ ions of montmorillonite in bentonite clay and two types of alkyl amines; hexadecylamine (primary amine) and hexadecyl trimethyl amine (tertiary amine). From the XRD analysis it was found that the primary amine-modified bentonite clay provided a greater inter-layer spacing than that with the tertiary amine-modified bentonite clay, although both were significantly higher when compared to the unmodified bentonite clay. The modified clay intercalated structure was then incorporated into a UV-curable coating formulation using epoxidized linseed oil acrylate as an oligomer to form a polymer clay nanocomposite. Energy consumption in the curing process and the physical properties of the cured films were studied as a function of the amount of modified clay incorporated into the coating formulations. The energy consumption in the curing process increased and hardness and flexibility of the cured films also improved.
\end{abstract}

Keywords: Nanocomposite coatings, UV curing, epoxidized oil acrylate, modified bentonite.

ENGINEERING JOURNAL Volume 16 Issue 3

Received 20 November 2011

Accepted 9 May 2012

Published 1 July 2012

Online at http://www.engj.org/

DOI:10.4186/ej.2012.16.3.13

This paper is based on the poster presentation at the German-Thai Symposium on Nanoscience and Nanotechnology 2011_Green Nanotechnology of the Future, GTSNN 2011, in Nakbon Ratchasima, Thailand, 13-16 September 2011. 


\section{Introduction}

Polymer/clay nanocomposites (PCNs) have emerged as new class of materials. PCNs typically exhibit exceptional increased mechanical and physical properties, such as a low gas permeability, increased swelling resistance, reduced flammability, compared to their micro- or macro-counterparts, and the virgin polymers $[1,2]$.

Recently, silicate type clays, such as montmorillonite, hectonite and saponite, have received a great deal of attention as reinforcing materials for polymers due to their potentially high aspect ratio and unique intercalation/exfoliation characteristics. The montmorillonite clay employed by the coatings industry is either hectorite or bentonite [3]. Montmorillonite (MMT) in form of bentonite clays are widely used in paint, particularly in interior water-based paint, due to their high affinity for water, where they function as viscosity modifiers. Therefore, bentonite clays are so-called montmorillonite (MMT). Since natural montmorillonites (Na-MMT) are filled with sodium cations, the hydrophilic properties are enhanced, hence leading to a high degree of swelling in water. This causes some difficulty in achieving a complete intercalation/exfoliation of the Na-MMT in the hydrophobic nature of most polymers. To produce an exfoliated nanocomposite, a high degree of wetting of polymer chains onto clay particles is required so that the polymer chains are exfoliated between the clay galleries. To achieve a better interaction between the clay particles and the organic polymers, the sodium cations present on the surface of Na-MMT are exchanged with organic molecules containing cationic groups, such as alkylammonium ions, to produce a cation modified montmorillonite (Mod-MMT) [4]. The obtained Mod-MMT has a more hydrophobic character and is incorporated into the thermoplastic polymer matrix to produce PCN materials. The incorporation of Mod-MMT into a polymer matrix was first disclosed by Nahin and Backlund of Union Oil Co. [5], and subsequentially PCNs have been shown to frequently exhibit better mechanical and chemical properties, compared with their macrocomposite counterparts $[6,7,8]$, due to the dimension and microstructure of the dispersed phase of the Mod-MMT, which significantly affects the properties of the PCNs.

UV-radiation curing is one of the most effective processes for rapidly transforming a liquid coating film into a solid film. This well-proven technology offers a number of advantages making it suitable for the preparation of composite polymers $[9,10]$, and has become attractive, especially in the paint, ink and coating industries, due to its fast curing time, which provides a very high productivity and a very low emission of volatile organic compounds. By adjustment of the light intensity, ultrafast curing can be carried out at the desired rate at ambient temperature [11].

Epoxidized linseed oil acrylate is a subclass of epoxy acrylate, and is one of the widely used resins in UV-curable coating systems. The resin has a low viscosity, low cost, good pigment wetting properties and provides relatively flexible coatings.

The objective of this research work was to study the effect of the amount and type of alkylamine used in the cationic exchange of monmorillonite modification upon the physical and mechanical properties of the modified monmorillonite. The modified monmorillonite was then incorporated into a UV curable coating formulation using epoxidized linseed oil acrylate as an oligomer to form the PCN. The physical properties of the dried UV curable coating films were tested.

\section{Experimental}

\subsection{Preparation of Modified Montmorillonite}

The two alkylamines, hexadecylamine (primary amine) and hexadecyl trimethyl amine (tertiary amine), were dissolved in $15 \mathrm{ml}$ distilled water and $1 \mathrm{ml} \mathrm{HCl}$ (conc.) to obtain three different concentrations of $1 \%, 2 \%$ and $3 \%(\mathrm{w} / \mathrm{w})$. Then, $2.5 \mathrm{~g}$ of montmorillonite dispersed in $100 \mathrm{ml}$ water was added to the appropriate alkyammonium solution and stirred for $1 \mathrm{hr}$. The white precipitate which had formed was then filtered and washed repeatedly to make it free from chloride ions. The resultant Mod-MMT was dried in an oven at 70 ${ }^{\circ} \mathrm{C}$ for $24 \mathrm{hr}$ before being ground into a fine powder and characterized. 


\subsection{Characterization}

\subsubsection{Infrared Spectroscopy (IR)}

The IR spectra of samples were recorded using a Nicolet Impact 400D infrared spectrometer with wave number varying from 600 to $4000 \mathrm{~cm}^{-1}$.

\subsubsection{X-Ray Diffraction (XRD)}

XRD- Rigaku Tint 2000 DMAX diffractometer, using $\mathrm{Cu} \mathrm{K} \alpha$ radiation, was used to obtain XRD patterns from which the crystal structure of the modified bentonite clay was evaluated. The interlayer spacing of the Mod-MMT was calculated according to Bragg's law.

\subsection{Modified Montmorillonite Application in UV-Curable Coating}

The obtained Mod-MMT bentonite clay was mixed in a UV-curable coating formulation using epoxidized linseed oil acrylate as the oligomer. Four different amounts (0, 0.5, 1 and 1.5\% (w/w)), of Mod-MMT were incorporated into the UV-curable coating formulations and then coated onto tin plates with a wet film thickness of $60 \mu \mathrm{m}$ and cured in the chamber. Energy consumption in the curing process was investigated using the UV-integrator and the physical properties of the dried films were tested.

\section{Results and Discussions}

\subsection{Modified Montmorillonite: Infrared Spectroscopy (IR)}

The preparation of the Mod-MMT was based on a cation-exchange reaction. The synthetic organophillic montmorillonites (OMMT) were made by the treatment of sodium montmorillonite (Na-MMT) with hexadecylamine (primary amine) or hexadecyl trimethyl amine (tertiary amine). The characterization of $\mathrm{Na}$ MMT and the corresponding OMMT was conducted using a Fourier Transform Infrared Spectrometer (FTIR). The results are shown in Fig. 1.

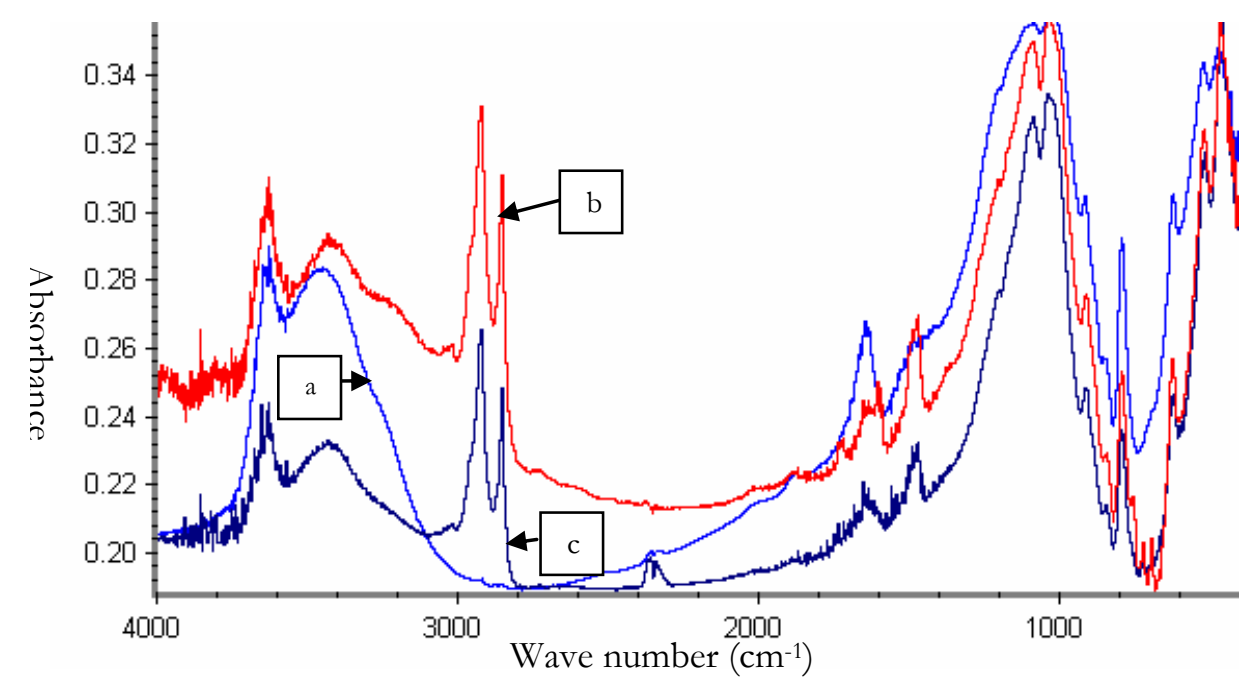

Fig 1. FT-IR spectra of (a) pure Na-MMT and (b) OMMT modified with hexadecyl trimethyl ammonium chloride at 3\% (w/w), (c) OMMT modified with hexadecyl amine at 3\% (w/w).

The FTIR spectra of Na-MMT and OMMTs show the presence of bands at about 3600 and $1100 \mathrm{~cm}^{-1}$ which are the typical hydroxyl group bands of Na-MMT (Fig. 1), while the spectra of both OMMTs show the presence of two new bands at about 2800 and $2900 \mathrm{~cm}^{-1}$, which are due to $\mathrm{C}-\mathrm{H}$ stretching of the 
aliphatic hydrocarbon of the alkyl chain [13]. This indicates that alkyl ammonium salts were incorporated into the lattice of MMT.

\subsection{X-Ray Diffraction (XRD)}

X-ray diffraction technique is used to identify the interlayer spacing of MMT and OMMT. The results of X-ray diffraction patterns of Na-MMT compare to that of the primary amine modified OMMT and the tertiary amine modified OMMT are shown in Fig. 2 and 3, respectively.

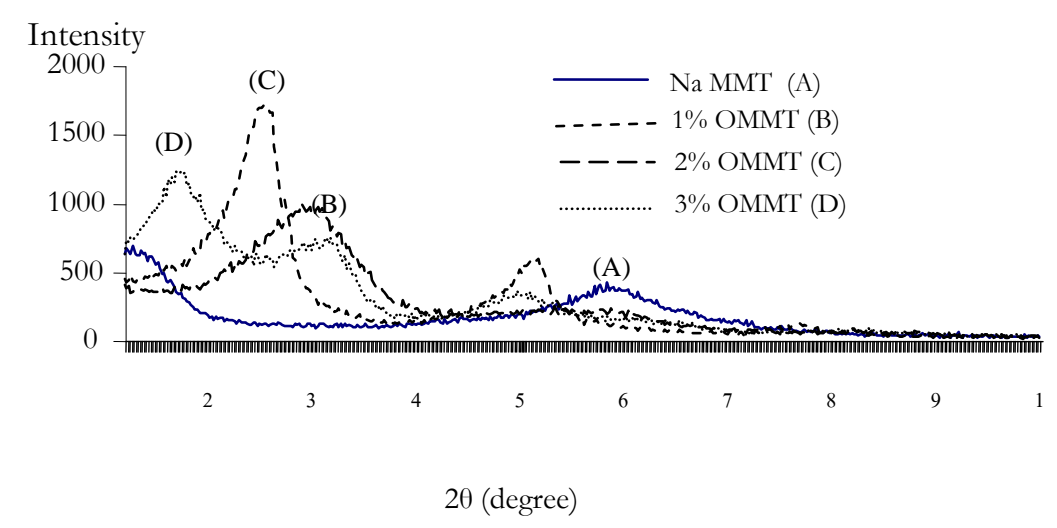

Fig. 2. XRD results of Na-MMT compared to that for the primary amine modified OMMT.

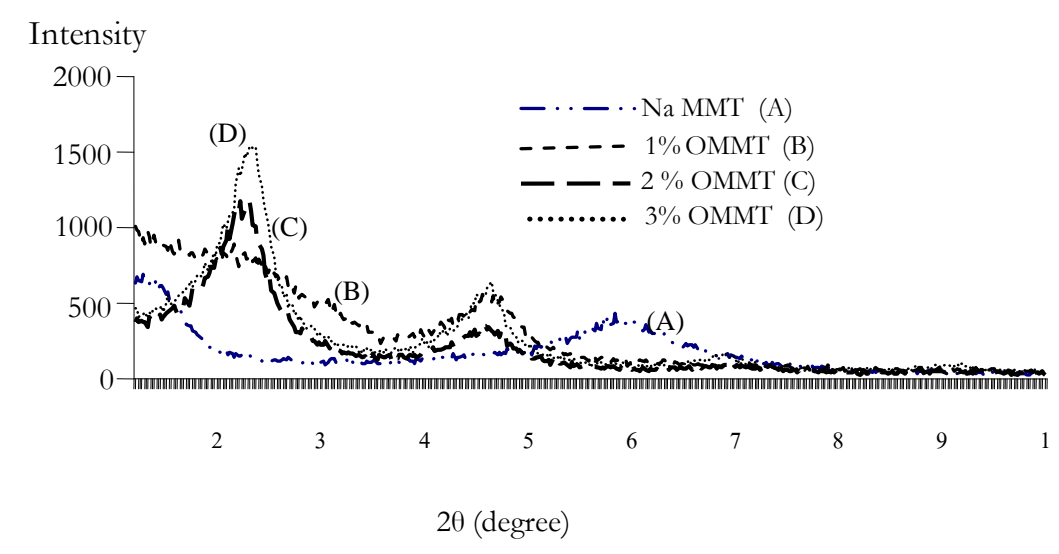

Fig. 3. XRD results of Na-MMT compared to that for the tertiary amine modified OMMT.

The peaks correspond to the (001) plane reflections of the clays. The averaged basal spacing (d-spacing) of Na-MMT and each OMMT were calculated by Bragg's law $(2 \mathrm{~d} \sin \theta=\mathrm{n} \lambda)$ [12], and were found to be $14.98 \AA$ at $2 \theta=5.9$ for Na-MMT.

The averaged basal spacing (d-spacing) of OMMT treated with 1\%,2\% and $3 \%(\mathrm{w} / \mathrm{w})$ of hexadecyl ammonium chloride (primary amine) and hexadecyl trimethyl ammonium chloride (tertiary amine) are shown in Fig. 4. 


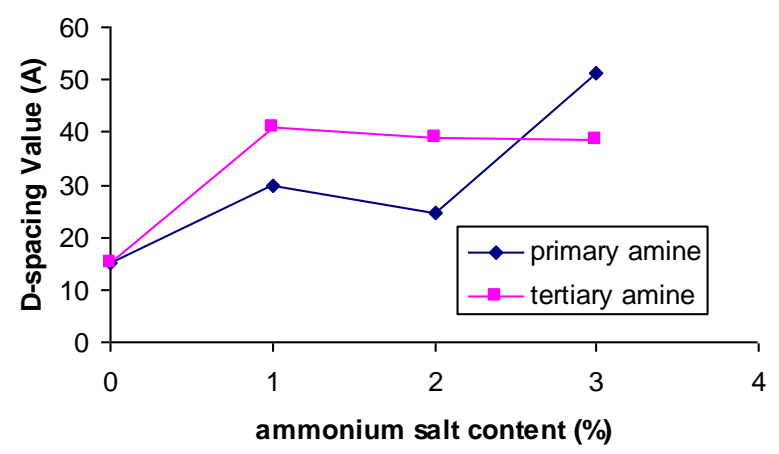

Fig. 4. Plot of D-spacing values of Na-MMT compared to OMMT modified with primary and tertiary ammonium salts.

The results indicated that the $\mathrm{Na}^{+}$ions in Na-MMT underwent cationic exchange with both ammonium salts. D-spacing values of OMMT modified with primary and tertiary ammonium salts increased compare to that of unmodified Na-MMT. However, the tertiary amine Mod-MMT provided higher $\mathrm{d}$-spacing values than that of $1 \%$ and $2 \%(\mathrm{w} / \mathrm{w})$ primary amine Mod-MMT. The highest $\mathrm{d}$-spacing value was observed with the $3 \%(\mathrm{w} / \mathrm{w})$ primary amine (hexadecylamine) Mod-MMT. The increment in the d-spacing derived from the long chain alkyl group of the exchanged hexadecylamine salt in the monmorillonite lattice. The greater the d-spacing obtained, the more each layer of clay structure separated from each other by long alkyl chain of ammonium salt. The clay structures are so-called intercalation. Thus, the 3\%(w/w) hexadecylamine Mod-MMT (OMMT) was selected to prepare the acrylic coating formulations.

\subsection{Application of Modified Montmorillonite in Coating Formulation}

The 3\% (w/w) hexadecylamine Mod-MMT (OMMT) was mixed into a coating formulation using epoxidized linseed oil acrylate as an oligomer. The coating formula was then coated onto a tin plate with a wet film thickness of $60 \mu \mathrm{m}$. The energy consumption in the drying process was measured, and the physical properties of the dried films were examined as various amounts of Mod-MMT in the formulation. The results are shown in Table 1.

Table 1. Results of physical properties and energy consumption in drying process.

\begin{tabular}{lcccc}
\hline \multirow{2}{*}{ Properties } & \multicolumn{5}{c}{ OMMT Contents (\%) } \\
\cline { 2 - 5 } & $\mathbf{0 . 0}$ & $\mathbf{0 . 5}$ & $\mathbf{1 . 0}$ & $\mathbf{1 . 5}$ \\
\hline Hardness (g) & 1000 & 1000 & 1500 & 2000 \\
Impact Resistance (lbs-inch) & 1600 & 1600 & 1600 & 1600 \\
Flexibility (mm) & 6 & 4 & 4 & 4 \\
Viscosity (cps) & 390 & 385 & 350 & 320 \\
Adhesion & 0 & $3 / 25$ & $4 / 25$ & $7 / 25$ \\
Gloss & 75.4 & 78.0 & 78.1 & 76.1 \\
Energy consumption in drying $\left(\mathrm{mJ} / \mathrm{cm}^{2}\right)$ & 228 & 310 & 396 & 542 \\
\hline Data are shown as the mean of 3 repeats. & & & &
\end{tabular}

The radiation energy consumption in drying of the coating films significantly increased as the amount of Mod-MMT in the formulation increased. The coating film incorporated with $1.5 \%$ Mod-MMT consumed UV radiation energy of $542 \mathrm{~mJ} / \mathrm{cm}^{2}$ relative to that of coating film with $0.5 \%$ Mod-MMT which consumed $310 \mathrm{~mJ} / \mathrm{cm}^{2}$. It showed that the greater the amount of Mod-MMT the higher the UV radiation energy is required to dry the coating film. This can be explained that the Mod-MMT particles could obstruct the UV radiation pathway to reach the photoinitiator molecules for photopolymerization causing more UV radiation energy is required to cure the coating film. 
As to the physical properties, the hardness of the films increased as the amount of modified clay in the coating formulation increased. This can be explained that the modified clay, with hydrophobic character from the long alkyl hydrocarbon chain of the hexadecyl ammonium salt provided good miscibility between the modified clay and the epoxidized linseed oil acrylate oligomer. As a result, the epoxidized linseed oil acrylate oligomer can diffuse into the spacing of the intercalation structure of the modified clay and enhanced the hardness of the coating films after the UV-radiation induced polymerization process was achieved. Likewise, the flexibility of the dried films increased slightly, due to the flexibility of oil acrylate oligomer used in the formulation.

The glossiness of the dried films decreased slightly as the amount of incorporated modified clay increased, especially at a concentration of $1.5 \%(\mathrm{w} / \mathrm{w})$. This was because the fine particles of modified clay might not truly disperse in the formulations causing the change in glossiness of the dried films. Moreover, the greater the amount of Mod-MMT added to the formulations, the lower the viscosity of the formulations obtained. This will be a benefit since higher contents of Mod-MMT can be added to obtain the desirable final film properties of the film without adversely affecting the viscosity of the coating formulations. The adhesion of dried film slightly decreased as the amount of Mod-MMT in the formulation increased. This was because the Mod-MMT interrupted the continuity of the film to the surface and caused the adhesion to decrease.

\section{Conclusion}

From this study, it can be concluded that the 3\%(w/w) primary amine (hexadecylamine) modified bentonite clay provided greater inter-layer spacing than that of tertiary amine (hexadecyl trimethyl amine). Selected Mod-MMT was incorporated into a UV-curable coating formulation using epoxidized linseed oil acrylate as an oligomer. The energy consumption in the curing process increased as a function of the amount of modified clay in the coating formulations increased. In addition, the hardness and flexibility of cured films also improved as the greater amount of modified clay was added in the formulations.

\section{References}

[1] E. M. Araujo, R. Barbosa, A. W. B. Rodrigues, T. J. A. Melo, and E. N. Ito, "Processing and characterization of polyethylene/Brazilian clay nanocomposites," Materials Science and Engineering., vol. 445, p. 141, 2007.

[2] R. Dagani, "Putting the nano into composites," Chemical and Engineering News., vol. 7, pp. 25, 1999.

[3] C. V. Hare, Protective Coatings: Fundamentals of Chemistry and Composition. Pittsburgh, PA: Technology Pub. Co., 1994, pp. 435.

[4] C. Chen, M. Khobaib, and D. Curliss, "Epoxy layered-silicate nanocomposites," Progress in Organic Coatings, vol. 47, pp. 376, 2003.

[5] P. G. Nahin and P. S. Backlund, "Organoclay-Polyolefin Compositions," U.S. Patent 3084117, Apr. 2, 1963.

[6] X. Fu and S. Qutubuddin, "Synthesis of polystyrene-clay nanocomposites," Materials Letters, vol. 42, pp. 12, 2000.

[7] J. Yeh, C. Chen, Y. Chen, C. Ma, K. Lee, Y. Wei, and S. Li, "Enhancement of corrosion protection effect of poly (o-ethoxyaniline) via the formation of poly (o-ethoxyaniline)-clay nanocomposite materials," Polymer, vol. 43, pp. 2729, 2002.

[8] L. E. Brus, W. L. Brown, R. P. Andres, R. S. Averback, W. A. Goddard, A. Kaldor, S. G. Louie, M. Moskovits, P. S. Peercy, S. J. Riley, R. W. Siegel, F. A. Spaepen, and Y. Wang, "Nanocomposites based on monmorillonite and unsaturated polyester," Journal of Materials Research, vol. 4, pp. 704, 1989.

[9] C. Decker, "Photoinitiated crosslinking polymerization," Progress in Polymer Science, vol. 21, pp. 593, 1996.

[10] C. Decker, "Kinetic study and new applications of UV radiation curing," Macromolecule Rapid Communication, vol. 23, pp. 1067, 2002.

[11] V. Shukla, M. Bajpai, D. K. Singh, M. Singh, and R. Shukla, "UV curing-Technical principle and mechanism", Ciba Specialty Chemicals, 2004.

[12] B. D. Cullity, Elements of X-ray Diffraction, 2nd ed. Reading, MA: Addison-Wesley, 1978, pp. 84.

[13] G. Socrates, Infrared Characteristic Group Frequencies. New York: Wiley, 1980. 